\title{
Brain Machine Interface
}

\author{
R. Kamalakannan ${ }^{1}$, N. Ravi Kumar ${ }^{2}$ \\ Assistant professor ${ }^{1,2}$, Department of EEE ${ }^{1,2}$, S.A. Engineering college ${ }^{1,2}$ Chennai $^{1,2}$, India $^{1,2}$
}

\begin{abstract}
In this project, the idea is to develop a prosthetic arm controlled by brain signals and MEMS, which will aid the difficulties faced by the physically challenged. The arm provided does the work of picking and placing a small object from one place to another. The arm movements are based on the brain signals, it pretends to do the actions like a natural human hand. The human brain produces different forms of electrical signals when subjected to a thought which are of different amplitudes and frequencies. These signals are basically classified into five different types and are called alpha, beta, gamma, delta and theta. These signals are retrieved by use of electrodes placed on the epidermal layer i.e., the outer skin layer of the skull. This method is called Electroencephalography (EEG) technique. After receiving signal from brain the signals are amplified using an Instrumentation amplifier, because the retrieved signal will be of very low amplitude. This amplified signal is compared with output of MEMS by AND logic. MEMS will detect head movement which is used to project arm in $x$ and $y$ direction. A photodiode sensor is used to grab the object and release it in targeted place.
\end{abstract}

Keyword: EEG, MEMS, LCD, ALE, IR, ADC.

\section{I.INTRODUCTION}

In recent times many research has been made and ongoing in interfacing human brain signals with machines as an advancement in technology to obtain automatic control or actions like natural human does and also for aiding human beings. In this paper, an attempt has been made to propose a thought controlled robotic arm, which uses the captured signals from the brain along with slight movement of head and processes it to control the robotic arm to perform actions like a normal human hand.

The human brain produces different forms of electrical signals when subjected to a thought which are of different amplitudes and frequencies. These signals are basically classified into five different types and are called alpha, beta, gamma, delta and theta [4]. These signals are retrieved by use of electrodes placed on the epidermal layer i.e., the outer skin layer of the skull. This method is called Electroencephalography (EEG) technique. An electroencephalogram detects abnormalities in the brain waves or electrical activity of the brain. During the procedure, electrodes consisting of small metal discs with thin wires are pasted on the scalp. The electrodes detect tiny electrical charges that result from the activity of the brain cells [3]. The charges are amplified and appear as a graph on a computer screen or as a recording. EEG technique deploys an electrode cap that is placed on the user's scalp specifically EEG placed on forehead for the acquisition of the brain signals. Controller which in turn operate the arm. The electrical activity of the brain can be monitored in real- time using an array of electrodes, which are placed on the scalp in a process known as electroencephalography. In order to bypass the peripheral nervous system, we need to find some reliable correlates in the brain signals that can be mapped to the intention to perform specific actions. Micro-Electro-Mechanical Systems, or MEMS, is a technology that in its most general form can be defined as miniaturized mechanical and electro-mechanical elements that are made using the techniques of micro fabrication. MEMS are an electrostatic transducer used for harvesting and converting the energy of vibrations or movement into electrical energy [2]. In this project, we are going to implement mind controlled robotic arm using EEG and MEMS. The EEG will monitor the brain activity and prosthetic arm will move according to the movement of the head with help of MEMS [5]. This prosthetic arm will aid people who are suffering from quadriplegia where they loss control over the body parts but with normal brain activity. Independent component analysis(ICA) was used to segregate obvious artifact EEG components from other sources, and a frequency-band representation was used to represent the sources computed by ICA. Examples of results include an $85 \%$ accuracy rate on differentiation between two tasks, using a segment of EEG only $0.05 \mathrm{~s}$ long and a $95 \%$ accuracy rate using a 0.5 -s-long segment [1].

\section{Existing System}

The existing system works completely using brain signals and processing it through neural networks which increases the complexity of the system and involves using of large number of electrodes which may range from 70 to 120 approximately [6]. Not only the system is complex but, the method of signal processing also tends to be difficult. This eventually brings about increased cost of the entire system, making it unaffordable to people of low economic status.

\section{Proposed System}

The proposed system focuses on the simplicity, methodology used and cost efficiency serving the same function as the existing system. To make it possible a modern day device called MEMS (Micro Electro Mechanical Systems) is used in combination with the EEG (Electro Encephalograph). The number of electrodes used is reduced to 3. A simple AND logic of outputs from MEMS and EEG is used to carry out the entire operation which is like the entire alternative of neural network analysis, thereby making the system in to simpler one [7]. 3. Advantages

- System that is being used here is simple in construction and in implementation.

- The BMI module employed in this project is Cost effective compared to other similar models that exist.

- $\quad$ The system being used has very high operating efficiency.

- Number of electrodes required is reduced to a small number viz. to 3 . 
- The proposed system is very reliable and accurate when compared to other systems. II BLOCK DIAGRAM DESCRIPTION

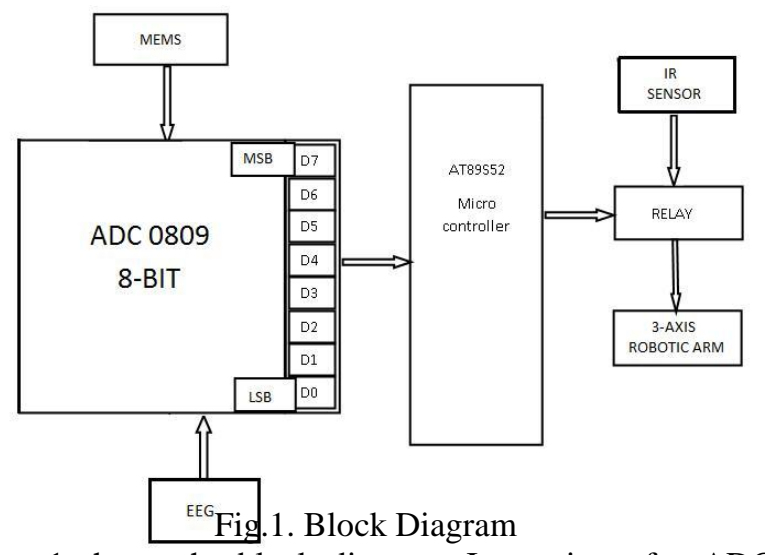

Figure1 shows the block diagram. It consists of a ADC where MEMS and EEG output is converted into digital signals which is given as input to microcontroller. Controller gives control signals to relay and inturn relay triggers the motor for movement of arm. Multichip modules require additional assembly steps. Yield is generally lower for multichip modules. Larger signals from the sensor are required to overcome the stray capacitance of the chip to chip interconnections, and stray fields necessitating a larger sensor structure. Larger packages are generally required to house the two-chip structure.

The latest generation ADXL2O2E is the result of almost a decade worth of experience building integrated MEMS accelerometers. It is the world's smallest mass-produced, low cost, integrated MEMS dual axis accelerometer. Polysilicon springs suspend the MEMS structure above the substrate such that the body of the sensor (also known as the proof mass) can move in the $\mathrm{X}$ and $\mathrm{Y}$ axes. Acceleration causes deflection of the proof mass from its center position. Around the four sides of the square proof mass are 32 sets of radial fingers. These fingers are positioned between plates that are fixed to the substrate. Each finger and pair of fixed plates make up a differential capacitor, and the deflection of the proof mass is determined by measuring the differential capacitance.

This sensing method has the ability of sensing both dynamic acceleration (i.e. shock or vibration) and static acceleration (i.e. inclination or gravity). The differential capacitance is measured using synchronous modulation/demodulation techniques. After amplification, the $\mathrm{X}$ and $\mathrm{Y}$ axis acceleration signals each go through a $32 \mathrm{KOhm}$ resistor to an output pin $(\mathrm{Cx}$ and $\mathrm{Cy})$ and a duty cycle modulator (the overall architecture can be seen in the block diagram in (Figure 3). The user may limit the bandwidth, and thereby lower the noise floor, by adding a capacitor at the $\mathrm{Cx}$ and $\mathrm{Cy}$ pin.An electroencephalogram detects abnormalities in the brain waves or electrical activity of the brain. During the procedure, electrodes consisting of small metal discs with thin wires are pasted on the scalp. The electrodes detect tiny electrical charges that result from the activity of the brain cells. The charges are amplified and appear as a graph on a computer screen or as a recording that may be printed out on paper. Your doctor then interprets the reading.

IR Sensors work by using a specific light sensor to detect a select light wavelength in the Infra-Red (IR) spectrum. By using an LED which produces light at the same wavelength as what the sensor is looking for, you can look at the intensity of the received light. When an object is close to the sensor, the light from the LED bounces off the object and into the light sensor. This results in a large jump in the intensity, which we already know can be detected using a threshold.

\section{SIMULATION RESULT}

The main purpose of using the microcontroller in our project is because high-performance CMOS 8-bit microcontroller with $8 \mathrm{~K}$ bytes of in-system programmable Flash memory. By combining a versatile 8-bit CPU with in-system programmable Flash on a monolithic chip, the Atmel AT89S52 is a powerful microcontroller which provides a highly-flexible and cost-effective solution to many embedded control applications. The programs of the microcontroller have been written in Embedded $\mathrm{C}$ language and were compiled using KEIL, a compiler used for microcontroller programming. The communication between PC and the microcontroller was established MAX 232 standard and those programs were also done in $\mathrm{C}$ language.The following programs are used at various stages for the mentioned functions. In this program, the various special function registers of the microcontroller are set such that they can send and receive data from the PC. This program uses the serial library to communicate with the ports. The figure 2 shows the commands used in the source code.

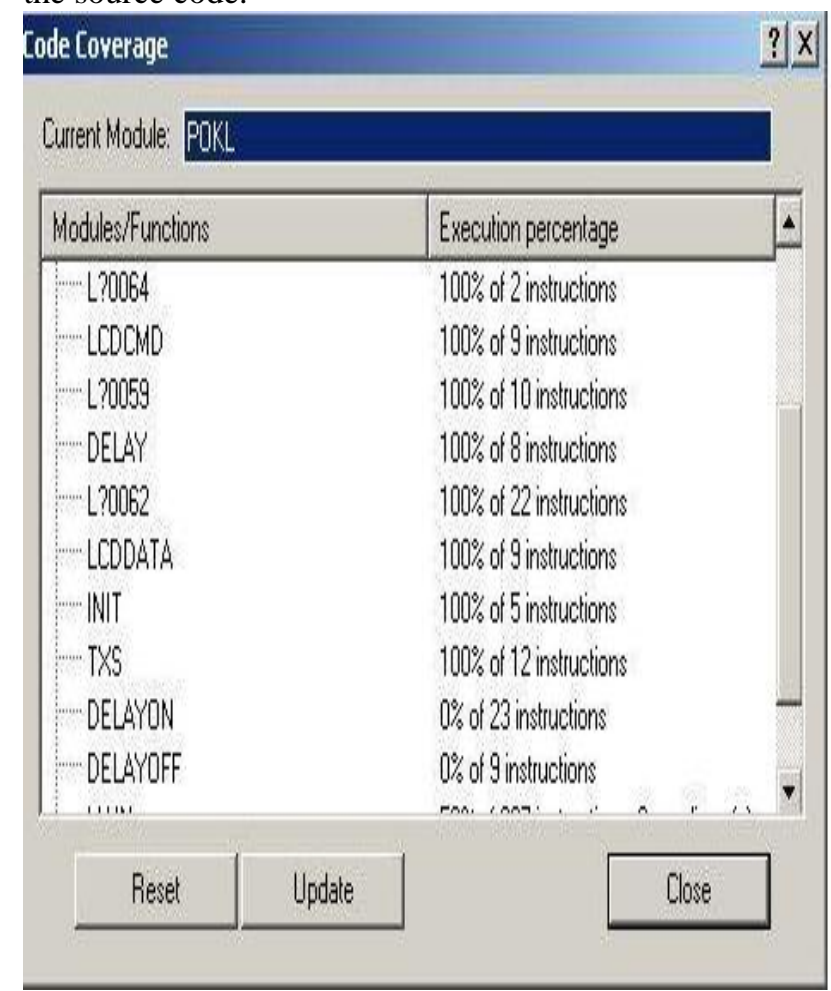

Fig.2. Simulation Result

The figure 3 shows the obtained debug results of the executed source code and the figure 4 shows the runtimes results using the keil compiler software. 


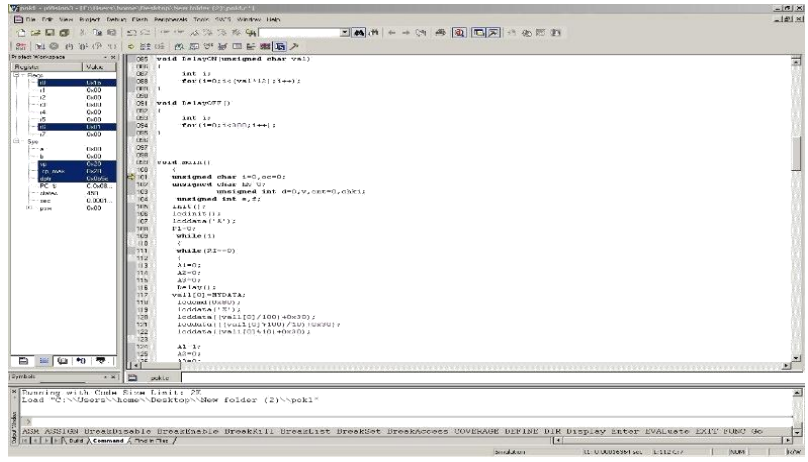

Fig.3. Program Result

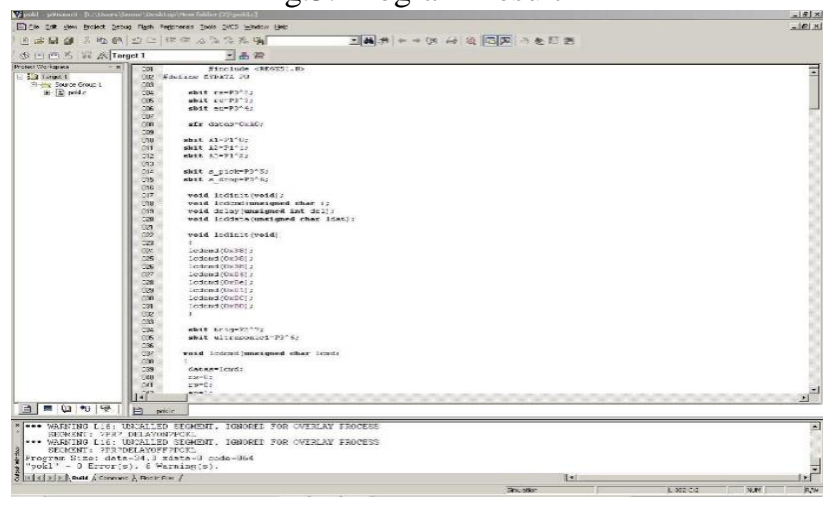

Fig. 4. Runtime Results

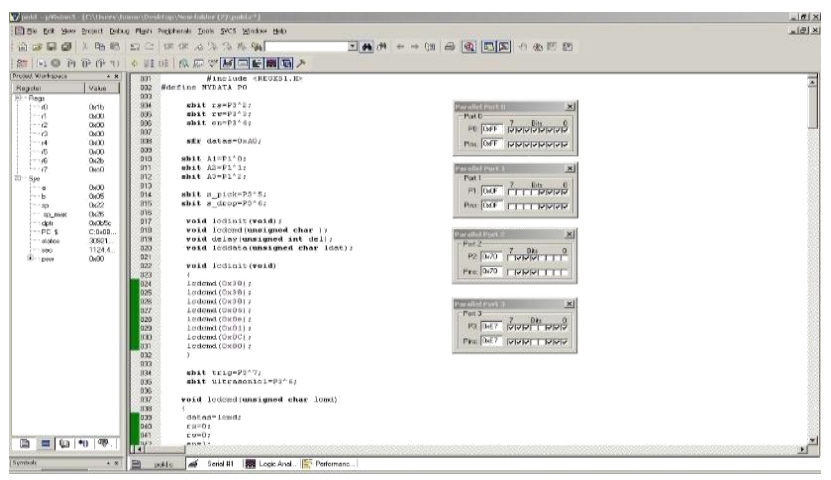

Fig.5.Output Window

The above figure 5 shows the output window along with the results of the executed source code in keil software.

\section{HARDWARE IMPLEMENTATION}

The figure 6 shows the hardware implementation. Basic setup behind this project started with the selection of components required to proceed with the experiment. Having decided with the components the next step was to assemble them and test their operation.

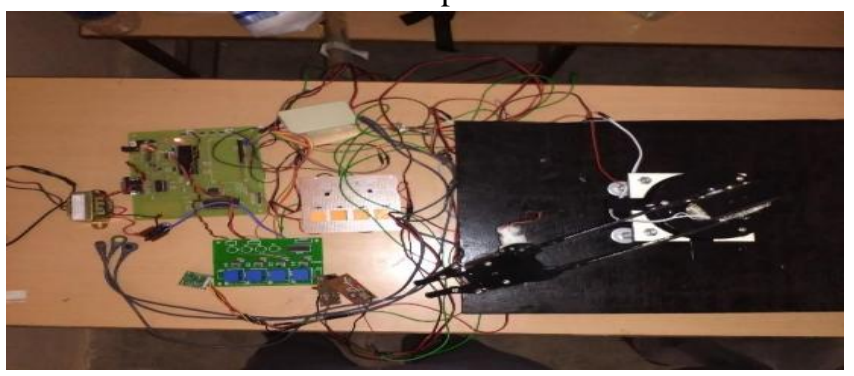

Fig. 6. Prototype Model

\section{CONCLUSION}

This chapter deals with hardware implementation and working picture of the kit. Photo describes the working prosthetic arm and main mother board where the components are assembled along with EEG electrodes. This implementation results in working model which can be used to aid quadriplegia patient. This kit is an effective alternate for their arm which does the actions of pic and place over in three dimension.

\section{REFERENCES}

[1] Benjamin J. Culpepper and Robert M. Keller, "Enabling Computer Decisions based on EEG Input", IEEE Transactions on Neural Systems and RehabilitationEngineering 11.4, December 2003

[2] C,I,Penaloza, Y,Mae, F.F.Culler, M.konjima, T.Arai, "Brain MachineInterface system Automation considering user preferences and error perception feedback," IEEE Transaction on Automatic Science and Engineering, August 2014

[3] J.Hokka, J.Raami, H.Hyvonen, M.Broas, J.Makkonen, J.Li, T.T.Mattila, M.Paulasto-Krockel, "Methods for reliability assessment of MEMS devices,'IEEE Electronic Components and Technology Conference(ECTC), August 2012

[4] Ling Li, Lei Xiao, and Long Chen, "Differences of EEG between Eyes- Open and Eyes-Closed States Based on Autoregressive Method", Journal of electronicscience and technology of China, vol.7, no.2, June 2009

[5] M.Mahmud, D. Hawelleck, A,Bertoldo, "EEG based brainmachine interface for navigation of robotic devices," IEEE RAS and EMBS Internation Conferenceon Biomedical Robotics and Biomechatronics, August 2013. [6] N.S.Kale, "MEMS Design, Fabrication and their application as Chemical andBiosensors," International Conference on VLSI Design(VLSID), August 2015 [7] S.Zhang, Y.Zeng, "Brain machine interface based on Diva model," Chineseconference on control and decision, August 2015

[8] Stancin, Tomazic, "Time- and computation-efficient calibrationof MEMS 3D accelerometers and gyroscopes," IEEE Transaction on sensors, August 2014

[9] T.Felzer, "Analysing EEG signals using the probability estimating guarded neural classifier," IEEE Transaction on Neural Systems and RehabilitationEngineering, December 2013 [10] Yuanqing Li, Chuanchu Wang, Haihong Zhang and Cuntai Guan, "AnEEG-based BCI System for 2D Cursor Control," IEEE International JointConference on Neural Networks, June 2008 